\title{
El 70 Festival de Cine de Berlín: entre chien et loup*
}

70th Berlin International Film Festival: entre chien et loup

Silvia Spitta ${ }^{\text {a }}$

DOI: https://doi.org/10.11144/Javeriana.cl25.fcbe

Dartmouth College, Estados Unidos

silvia.spitta@dartmouth.edu

ORCID: https://orcid.org/0000-0002-7372-2982

Recibido: 10 Enero 2021

Aceptado: 20 Agosto 2021

Publicado: 22 Noviembre 2021

La Berlinale 2020 que se celebró este año había sido particularmente anticipada porque coincidían en ella grandes celebraciones e importantes cambios de dirección. El festival no solo cumplía 70 años desde su fundación, sino que también se jubilaba su reconocido director Dieter Kosslick después de 18 años durante los cuales le dio fama internacional, transformándolo en uno de los festivales de cine más importantes del mundo. A la vez, se le daba la bienvenida a los nuevos codirectores Mariette Rissenbeek — socióloga con formación teatral, especialista en relaciones públicas y recientemente productora de películas para la televisión - y Carlo Chatrian - conocido director del Festival de Cine Independiente de Locarno-. Rissenbeek y Chatrian entraron dándole un nuevo aire al festival, acortando la lista de ciclos paralelos y creando una sección dedicada a promover películas arriesgadas y estéticamente innovadoras. Como si esto fuera poco, también se celebraban los 50 años de la importante sección Fórum, dedicada a la proyección y estudio de formas cinemáticas alternativas y a la exploración de nuevas y arriesgas propuestas estéticas, y se le daba la bienvenida a la nueva directora del festival, Cristina Nord.

Estos aniversarios, despedidas y bienvenidas tan anticipados tuvieron lugar bajo el acecho de la sombra del COVID-19 y la pregunta de si llegarían los equipos de la China y del resto del mundo (muchos no llegaron). También se vieron sacudidos por un atentado neonazi en la ciudad de Hanau el día antes de la inauguración del festival y por el escándalo causado por la revelación de que Alfred Bauer, que había sido un director del festival y en honor a quien se había nombrado uno de los premios más prestigiosos, había estado en la cúpula del nazismo — algo que hasta ahora había pasado desapercibido aún por los historiadores del festival—. El premio fue abolido inmediatamente y reemplazado por un nuevo Oso de Plata que le fue otorgado a la única comedia de la competición, Effacer l'historique (Francia), de Benoît Delépine y Gustave Kervern.

La Berlinale 2020 se vio enmarcada por el estreno del importante documental de Aysun Bademsoy Traces ('huellas') que retrata las vidas de los familiares de las víctimas turco-alemanas que fueron asesinadas por un miembro del grupo terrorista neonazi Nationalsozialistischer Untergrund (NSU) y que vieron frustrada la justicia por el racismo de la policía alemana que insistía que los crímenes habían sido cometidos por miembros de la comunidad turca. Este pre-juicio hizo que el proceso tardara años hasta que finalmente los policías dieron con la banda minúscula de terroristas que iba de ciudad en ciudad a través del país matando indiscriminadamente a pequeños comerciantes, tenderos, costureras y otros miembros de la comunidad turco-alemana. El documental se mostró unos días antes de la inauguración oficial del festival, algo que resultó casi clarividente, ya que en la víspera de la inauguración ocurrió el grave ataque neonazi en el que un individuo asaltó dos bares de shisha - frecuentados muchas veces por jóvenes descendientes de turcos o árabes-, matando a nueve personas. Las denuncias de este atroz crimen por parte de la Berlinale, los actores y políticos como la ministra de cultura, Monika Grütters, no se hizo esperar. En la alfombra roja, la noche de la

Notas de autor

\footnotetext{
a Autora de correspondencia. Correo electrónico: silvia.spitta@dartmouth.edu
} 
inauguración, Maryam Zaree, miembro del jurado de la sección Amnesty International del festival, organizó una protesta. Esta reconocida actriz había estrenado el documental Born in Evin en la Berlinale el año pasado. Hija de prisioneros políticos, Zaree nació en una cárcel en Teherán y su documental retrata y denuncia los abusos cometidos por el régimen iraní. También actúo en la película Undine, estrenada en esta Berlinale y muy celebrada, del director Christian Petzold, esposo de Aysun Bademsoy.

Pero allí no terminó el protagonismo de Irán. There Is No Evil del iraní Mohammad Rasoulof ganó el Oso de Oro a la mejor película. Con esta decisión, el jurado presidido por Jeremy Irons repitió la conocida solidaridad política de la Berlinale con Irán y la persecución de los artistas, dejando de lado los trabajos maravillosos de las estadounidenses Kelly Reichardt (First Cow) y Eliza Hittman (Never Rarely Sometimes Always), esta última película se llevó el Gran Premio del Jurado. En la película de Hittman, la dificultad y el dolor que conllevan abortar en EE. UU. son contados a través de las experiencias de una adolescente de un pequeño pueblo que viaja con su prima a Nueva York para poder realizar la operación. El filme trata este tema tan delicado en la política estadounidense a través del frecuente uso del primer plano, enfocando minuciosamente las expresiones de las chicas, su confusión, sufrimiento y las constantes vejaciones machistas que aguantan. En la entrega de premios, con el galardón en la mano, Hittman lo dedicó a "los trabajadores sociales y los médicos que protegen la vida y los derechos de esas mujeres".

La presencia latinoamericana también se vio con la participación de Kleber Mendonça Filho (O Som ao Redor [2012], Aquarius [2016] y Bacurau [2019]) y de Bérénice Bejo (The Artists [2011], Le Passé [2013], The Childhood of a Leader [2015], Eternité [2016] y After Love [2016]) en el prestigioso jurado internacional de la competencia que reparte el Oso de Oro, así como con la participación de la chilena Dominga Sotomayor, la primera mujer en obtener el premio a la mejor dirección por Tarde para morir joven en el Festival de Cine de Locarno, que participó en el jurado de la nueva y prestigiosa sección Encuentros, promovida por Chatrian.

La sección del Fórum dedicada a las grandes colaboraciones y las estéticas innovadoras en el cine celebró sus 50 años estrenado El tango del viudo y su espejo deformante, de Raúl Ruiz y Valeria Sarmiento, en la ceremonia de apertura. El Fórum no pudo haber escogido un mejor ejemplo de la inmensa labor de rescate, trabajo de archivo, reconstrucción y publicitación del cine experimental y/o estéticamente innovador que la obra de Raúl Ruiz, quien había tenido una importante presencia en esa sección a lo largo de los años y que al morir, hace casi diez años, dejó un corpus de aproximadamente cien películas, así como varias que quedaron inconclusas cuando tuvo que huir del régimen de Pinochet para exiliarse en Francia. Muchas de ellas están ahora en un archivo de la Universidad de Duke, donde se están digitalizando y preservando con el fin de ser repatriadas en el 2021 para coincidir con el décimo aniversario de la muerte del gran cineasta. El tango del viudo y su espejo deformante forma parte de la trilogía del realismo socialista, obra fundamental en la historia del cine mundial, en la que está trabajando la codirectora y viuda de Raúl Ruiz, Valeria Sarmiento, con el apoyo de Poetastros, un gran equipo de restauración y producción encabezado por Chamila Rodríguez y que incluye al sonidista Galut Alarcón, y las composiciones (con sierras de mano y theremín para crear un ambiente de horror) de Jorge Arriagada. La película había quedado inconclusa y sin sonido y el equipo encontró solo seis de sus siete rollos. Para reconstruir los diálogos, el equipo contrató a varias mujeres sordas profundas (es decir que nacieron sin oír) para leer los labios de los actores. Valeria Sarmiento tuvo la brillante idea de reconstruir la película honrando la poética de Ruiz, quien, como es conocido, pensaba que el cine es un espejo dotado de memoria hecho de partes que se reflejan entre sí. Por eso Sarmiento, como homenaje a Raúl Ruiz, decidió que la segunda parte repitiera la primera, pero al revés, y, de esta manera, podía simultáneamente repetir y distorsionar, creando dentro del mismo filme el reflejo del reflejo. La primera película de la trilogía, La telenovela errante, ya ha sido restaurada y fue estrenada en Locarno en el 2017. La trilogía va a culminar con Realismo socialista como una de las Bellas Artes, que actualmente está siendo restaurada, para ser estrenada en el 2021 y coincidir con el décimo aniversario de la muerte de Raúl Ruiz.

Otra película muy celebrada que jugó un importante papel en esta Berlinale fue Los Lobos de Samuel Kishi Leopo (México) que ganó el gran premio en la sección Generación Kplus del festival, además del Premio de 
la Paz. Esta fue la película escogida por la Berlinale para promover el cine entre los escolares de la ciudad, que obtuvieron entradas gratis y llenaron las funciones con gran algarabía y conversaron animadamente con el director y la protagonista principal al final de las varias funciones. Con el lema "Ustedes son fuertes como los lobos. Los lobos no lloran. Los lobos muerden. Aúllan. Y protegen sus hogares” la película se dirigió a los jóvenes al retratar a dos niños Max, de 8 años, y su hermano menor Leo, que viven con su madre Lucía (Marta Reyes Arias) y que han cruzado la frontera México-EE. UU. en busca de una vida mejor, pero que se encuentran en situaciones extremamente precarias cuando llegan a Estados Unidos. Ya que no tienen a nadie en ese país, la madre los encierra en el cuartucho donde viven cuando sale a trabajar. Al comienzo, los chicos tienen miedo de su nuevo entorno y la obedecen. Encerrados todo el día pasan el tiempo usando las paredes del cuarto como una pizarra en la que proyectan sus deseos, sueños y el terror que sienten, pero poco a poco empiezan a salir y conocer el mundo a su alrededor. Cuando esta película se muestre en EE. UU. va a causar sensación, dada la miseria humana causada por el Gobierno de Trump y su política de separación de padres y niños migrantes a modo de medida "disuasoria" del flujo migratorio.

El silencio del rio, un cortometraje de la peruana Francesca Canepa, plantea una reflexión poética sobre la naturaleza del ser humano y de la Amazonía, también se enfoca en los mundos oníricos de niños que enfrentan la incertidumbre, el desarraigo y la soledad. El joven protagonista, Juan, siguiendo la tradición familiar, quiere convertirse en el nuevo orador del pueblo e intentar así pasar más tiempo con su padre. En el 2017 Canepa ya había dirigido el cortometraje Aya, que se estrenó en Cannes y que narra la historia de un niño quechua hablante que trabaja en la salinera de Maras, en Cusco, para ahorrar dinero para poder enterrar a su mamá, con cuya muerte ya ha soñado. Otras películas dirigidas a niños mayores de 9 años en la sección KPlus fueron El nombre del hijo de la argentina Martina Matzkin, que fue también muy celebrada y obtuvo una mención especial en Generación Kplus y además ganó el Oso de Cristal al mejor cortometraje. Esta película narra la historia de un joven que recién está entrando en la adolescencia y a quien muchos confunden con una niña. Él logra superar sus dudas sobre su sexualidad gracias a una estrecha relación con su padre. Otra de las películas fue Mamá, mamá, mamá de la argentina Sol Berruezo Pichon-Rivière que se enfoca en una niña (finalmente) que sobrevive a la muerte de su hermanita y la crisis de su madre. Esta película muestra con un detalle maravilloso cómo la familia empieza a llenar de luz el vacío y la tristeza que dejó la muerte. 


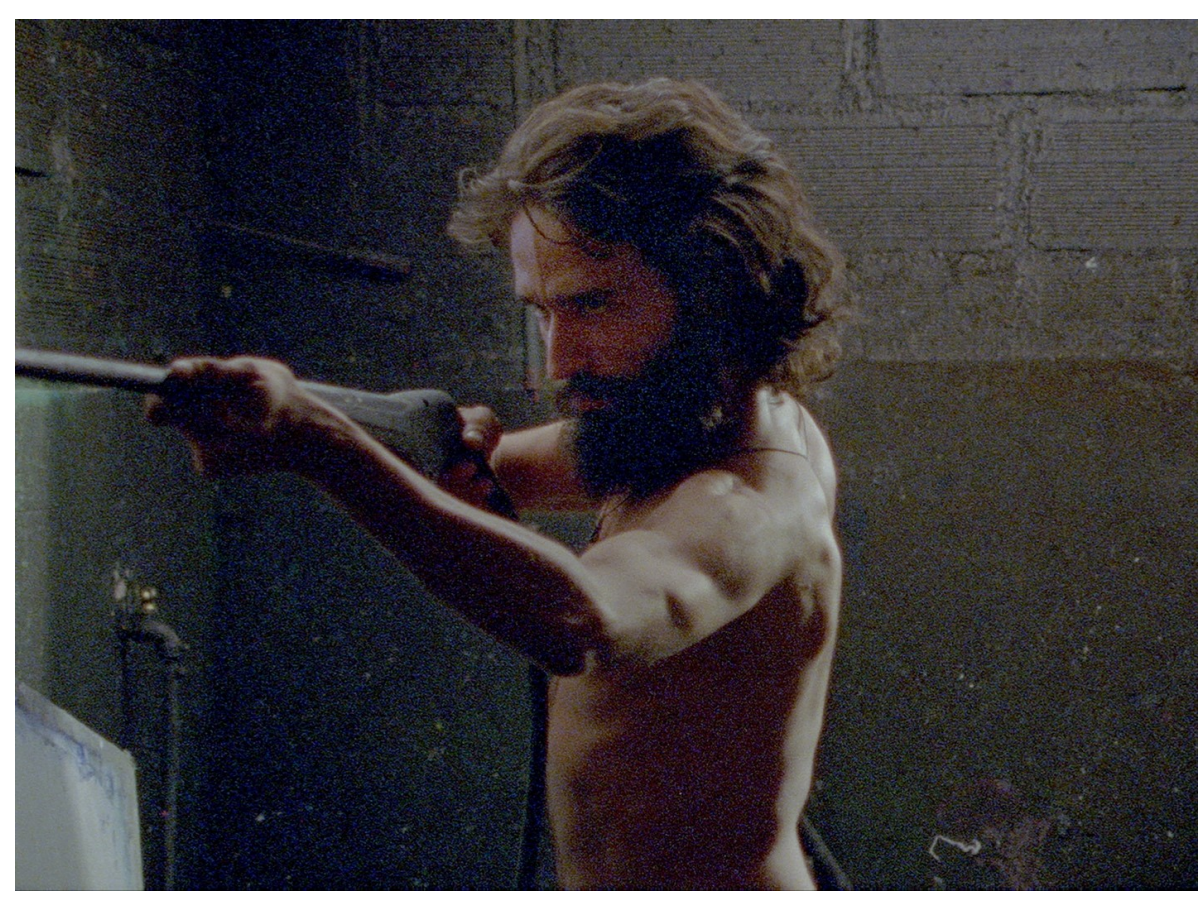

FIGURA 1.

Pinky (Luis Felipe Lozano). Camilo Restrepo, Los conductos

Fuente: cortesía Festival Internacional de Cine de Berlín

Los conductos del colombiano Camilo Restrepo ganó el premio a la Mejor Ópera Prima. Nacido en Medellín en 1975, época en que la ciudad experimentó la peor violencia, Restrepo viajó a París a estudiar arte. La mayor parte de su obra (filmada y financiado por él mismo) se enfoca alegóricamente en la historia de la violencia de su país, retratada a través de los ojos de jóvenes de la calle que se encuentran en situaciones de gran precariedad y que sobreviven solo gracias a su dedicación al arte. Los tatuajes, el grafiti y el punk constituyen el trasfondo de sus cortometrajes a la vez alegóricos y apocalípticos. En Los conductos, el protagonista Pinky (Figura 1), un drogadicto visionario, huye del líder de una secta por calles abandonadas y una ciudad en llamas, y se refugia en una fábrica ilegal de camisetas poblada de fantasmas. Filmada en $16 \mathrm{~mm}$, esta ópera prima culmina años de trabajo durante los cuales Restrepo solo hizo algunos cortometrajes ( 80 minutos en total): Tropic Pocket (2011), Cómo crece la sombra cuando el sol declina (2014), La Bouche (2017) — que se estrenó en la Quincena de Realizadores de Cannes en el 2017-y los ganadores del Pardino d'argento en el Festival de Cine de Locarno, La impresión de una guerra (2015) y Cilaos (2016).

Como ya es de esperarse, el cine documental hispano se encontró muy bien representado. Irene Gutiérrez (Hotel Nueva Isla [2014], Diarios del exilio [2019]) estrenó Entre perro y lobo, también en la sección Fórum. El título le hace eco a la expresión en francés "entre chien et loup", que designa el momento durante el atardecer cuando es difícil distinguir las cosas. Antiguamente los pastores no sabían si la forma que veían acercarse era la del perro que protegía al rebaño o la del lobo que lo iba a atacar. El documental de Gutiérrez retrata a los soldados rasos cubanos veteranos de la guerra de Angola que regresaron traumados a sus pueblos en la Sierra Maestra, pero que siguen en pie de guerra listos para defender el país a pesar de que el Gobierno nunca celebró sus hazañas. Allí retomaron la vida que habían dejado atrás cuando fueron a la guerra y volvieron a cultivar el campo. Recordándonos el teatro-documental de Teatro de guerra de Lola Arias, estrenado en la Berlinale el año pasado, en Entre perro y lobo los soldados recuerdan, rememoran y re-representan sus vivencias de la guerra, escenificándolas de diversas maneras en la Sierra Maestra como si estuvieran actuando en el teatro. A pesar de que están solos y nadie les ayuda a bregar con el estrés postraumático del que sufren, Estebita, Alberto y Miguel (Figura 2) emergen de las sombras en las que los ha dejado abandonados el Estado cubano. 


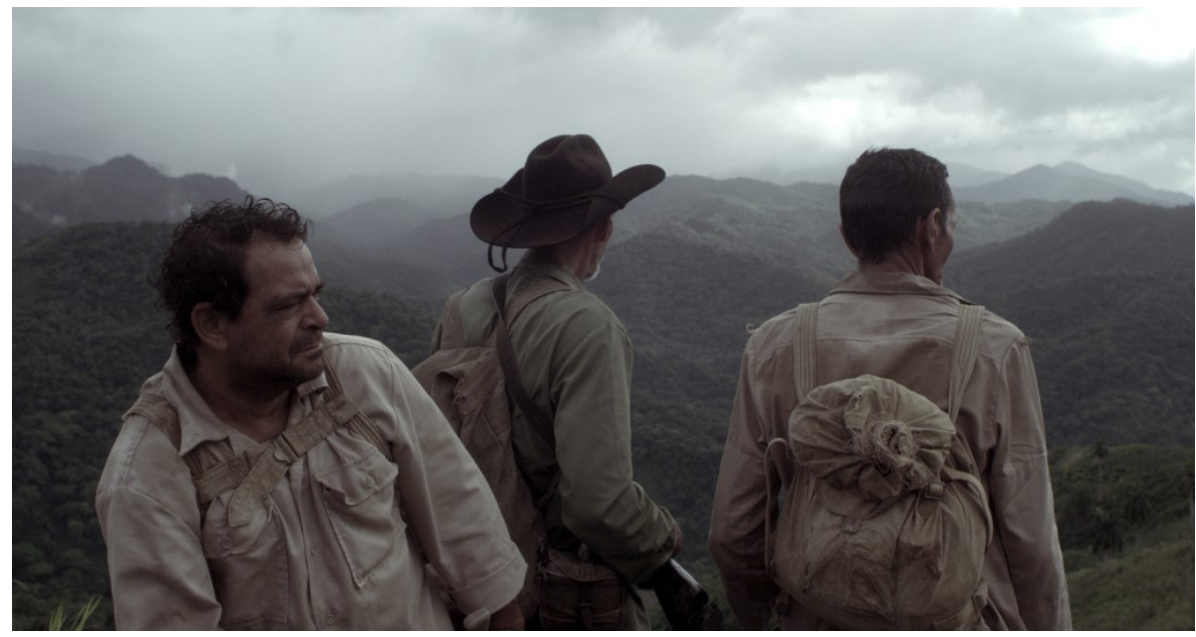

FIGURA 2.

Juan Bautista López, Miguel Soto, Alberto Santana. Irene Gutiérrez, Entre perro y lobo Fuente: cortesía Festival Internacional de Cine de Berlín

Gutiérrez, cineasta nacida en Ceuta, hizo su doctorado y enseñó cine en la Escuela Internacional de Cine y TV (EICTV) ubicada en San Antonio de los Baños, Cuba, y este documental fue su tesis doctoral. ${ }^{1} \mathrm{Su}$ próximo proyecto, Orfeo en la frontera, va a documentar las vidas de las mujeres que pasan por Ceuta camino a Europa en busca de asilo.

Anunciaron tormenta de Javier Fernández Vázquez (Bilbao, 1980) trata de una de las últimas incursiones (y masacres) españolas en la isla de Fernando Poo (territorio perteneciente a la actual Guinea Ecuatorial) en lo que restaba de su vasto imperio. Ësáasi Eweera, uno de los últimos líderes bubis que se oponía a la presencia española, fue detenido y su aldea arrasada. Esta historia es reconstruida a través de lecturas en voz alta de los documentos del Archivo General de la Administración que se encuentran en Madrid, y de la memoria de los pocos sobrevivientes que narran la historia oral del pueblo. Este contrapunteo entre documento real legal e historia oral resalta el hecho de que los documentos, supuestamente fidedignos, son en efecto tergiversaciones minuciosamente construidas para velar los verdaderos hechos y la rapiña española. En lugar de reafirmar la pátina imperial de proyecto "civilizatorio" y evangelizador que dominó el imaginario y la retórica imperiales, el filme demuestra cómo el Imperio español, además de violento, fue un imperio basado en la mentira y la violencia de la letra. 


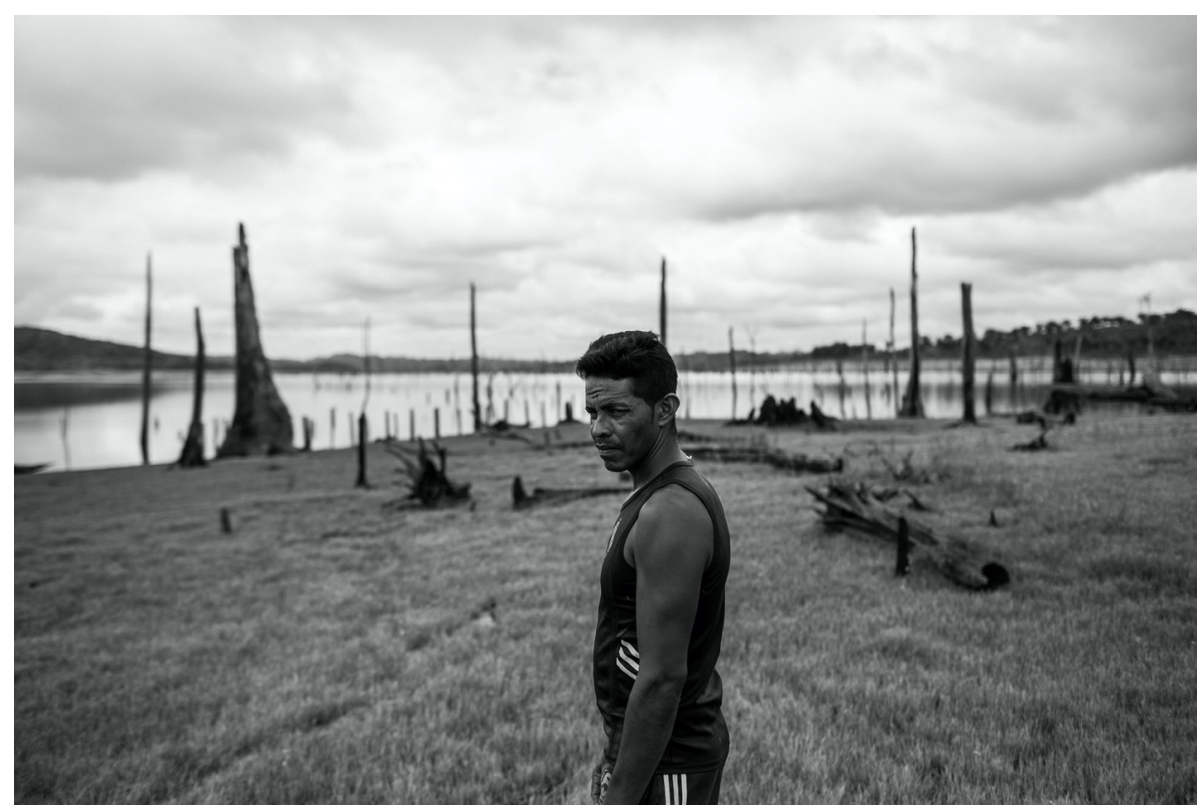

FIGURA 3.

O reflexo do lago, Fernando Segtowick

Fuente: cortesía Festival Internacional de Cine de Berlín

Nominada para el premio al mejor documental, $O$ reflexo do lago (Figura 3), del brasileño Fernando Segtowick, es una reflexión poética sobre el ecocidio de la Amazonía que se ha acelerado con Jair Bolsonaro, causando consternación mundial. Centrándose en los pobladores que perdieron sus pueblos y viviendas en 1980 cuando se construyó Tucuruí, una de las más grandes represas y centrales hidroeléctricas en el mundo, $O$ reflexo do lago muestra cómo el neoliberalismo beneficia solo a unos pocos, y sigue el empobrecido día a día de algunos de los pobladores que fueron desplazados cuando se construyó la hidroeléctrica. Inspirado en las fotografías de Paula Sampaio O Lago do esquecimento, la película es filmada en blanco y negro y se detiene fotográficamente en algunos árboles que rompen la superficie del lago como los tétricos esqueletos en el libro de Sampaio. En su lancha, una señora reflexiona que el lago por el que está navegando antes era una maravillosa selva. Este primer largometraje de Segtowick subraya la crítica mundial al filmicidio que está efectuando Bolsonaro con su política de destrucción cultural - filmicido que va de la mano del ecocidio que está perpetrando-. La película termina también haciendo alusión a esta desastrosa política con espectaculares tomas de los criminales incendios en la Amazonía que se ven en las noticias desde que Bolsonaro llegó al poder.

El documental Responsabilidad empresarial del argentino Jonathan Perel, su primer largometraje, también es una reflexión de las desastrosas políticas económicas neoliberales establecidas en Latinoamérica en los años 80 y cuyas secuelas estamos viviendo hoy en día. Perel se basa en extensas investigaciones y documentaciones sobre la complicidad de las grandes empresas, tanto multinacionales como nacionales, y en la violencia y represión de la dictadura militar de los años 80, con la cual se instauró el neoliberalismo en Argentina. Este documental muestra, por un lado, cómo esas empresas se beneficiaron en esos años siguiendo políticas antilaborales y sistemáticamente silenciando, marginando y "desapareciendo" a trabajadores y organizadores sindicales, y, por el otro, que, a pesar de sus abusos, siguen operando impunemente hoy en día. La voz en off del director se erige como un memorial de agravios; su interminable lista de los crímenes cometidos acompaña largas tomas de las fachadas de diversas empresas que colaboraron con la dictadura. Tal como lo señala el título, en Responsabilidad empresarial los crímenes cometidos por las grandes empresas durante la dictadura emergen de las sombras y ellas son declaradas responsables. El documental se erige como un único juicio. 
En esta Berlinale volvimos a presenciar la creciente importancia de lo que llamé anteriormente neodocumentalismo en el cine hispano. Esta vez, muchas de las películas que fueron estrenadas se debatían entre chien et loup, como si el neodocumentalismo latinoamericano se hubiera traslapado a todo el cine. Así Los Lobos, Los conductos y tantos otros largometrajes, a pesar de no ser documentales, se pueden ver en clave documental y los documentales como Entre perro y lobo dependen de escenificaciones teatrales para ser documentales. A pesar de los grandes debates políticos, y el acecho del COVID-19, entonces, la Berlinale 2020 cerró apostando por la solidaridad y celebrando sus 70 años —además de los 50 años del Fórum-, y, de esta manera, celebrando también las grandes colaboraciones entre la vida y el cine, el arte y la política.

\section{Notas}

* Sección Pliegos sueltos

1 La EICTV es una organización no gubernamental inaugurada el 15 de diciembre de 1986, siendo sus figuras fundadoras: Gabriel García Márquez, Fidel Castro Ruz, Fernando Birri y Julio García Espinosa. Nace bajo el auspicio de la Fundación del Nuevo Cine Latinoamericano en conjunto con el Estado cubano.

\section{Licencia Creative Commons CC BY 4.0}

Cómo citar: Spitta, Silvia. "El 70 Festival de Cine de Berlín: entre chien et loup”. Cuadernos de Literatura, vol. 25, 2021, pp. 1-10, https://doi.org/10.11144/Javeriana.cl25.fcbe 